\title{
Revisión sistemática y metaanálisis sobre la prevalencia de depresión, ansiedad e insomnio en trabajadores sanitarios durante la pandemia de COVID-19
}

\section{Prevalence of depression, anxiety, and insomnia among healthcare workers during the COVID-19 pandemic: A systematic review and meta-analysis}

\author{
Alba de Juan Pérez ${ }^{1}$ \\ ${ }^{1}$ Hospital Universitario de San Juan, Alicante, España.
}

\section{Resumen}

Este trabajo es un comentario del artículo: Pappa S, Ntella V, Giannakas T, Giannakoulis VG, Papoutsi E, Katsaounou P. Prevalence of depression, anxiety, and insomnia among healthcare workers during the COVID-19 pandemic: A systematic review and meta-analysis. Brain Behav Immun. 2020 Aug;88:901-907. doi: 10.1016/j.bbi.2020.05.026.

\section{Abstract}

This text is a commentary on the article: Pappa S, Ntella V, Giannakas T, Giannakoulis VG, Papoutsi E, Katsaounou P. Prevalence of depression, anxiety, and insomnia among healthcare workers during the COVID-19 pandemic: A systematic review and meta-analysis. Brain Behav Immun. 2020 Aug;88:901-907. doi: 10.1016/j.bbi.2020.05.026.

Sección coordinada por

Consol Serra (consol.serra@upf.edu) I Ma del Mar Seguí (mm.segui@ua.es)

Fechas · Dates

Recibido: 2021.06.05 Aceptado: 2021.06 .23

Publicado: 2021.07.15

\section{Correspondencia $\cdot$ Corresponding Author}

Alba de Juan Pérez

Correo electrónico: dejuan_albper@gva.es 


\section{Traducción del resumen del artículo comentado}

Antecedentes: La pandemia de COVID-19 ha tenido el potencial de afectar significativamente la salud mental de los trabajadores de la salud, que se encuentran en la primera línea de esta crisis. Por lo tanto, es una prioridad inmediata monitorizar el estado de ánimo, sueño y otros problemas de salud mental para comprender los factores mediadores y dirigir las intervenciones personalizadas. El objetivo de esta revisión es sintetizar y analizar la evidencia existente sobre la prevalencia de depresión, ansiedad e insomnio entre los trabajadores sanitarios durante el brote de COVID-19.

Métodos: Se realizó una búsqueda sistemática en bases de datos bibliográficas hasta el 17 de abril de 2020. Dos revisores evaluaron de forma independiente los artículos a texto completo de acuerdo con unos criterios predefinidos. Se evaluó el riesgo de sesgo para cada estudio individual y se agruparon los datos mediante metaanálisis de efectos aleatorios para estimar la prevalencia de problemas específicos de salud mental. El protocolo de revisión está registrado en PROSPERO y está disponible en línea.

Hallazgos: Se incluyeron 13 estudios en el análisis con un total combinado de 33062 participantes. La ansiedad se evaluó en 12 estudios, con una prevalencia combinada del 23.2\% y la depresión en 10 estudios, con una tasa de prevalencia del 22.8\%. Un análisis de subgrupos reveló diferencias ocupacionales y de género en el personal sanitario femenino y en enfermeras, que exhiben tasas más altas de síntomas afectivos (ansiedad y depresión) en comparación con el personal masculino y médicos, respectivamente. Finalmente, la prevalencia del insomnio se estimó en 38.9\% en 5 estudios.

Interpretación: La evidencia preliminar sugiere que una proporción considerable de trabajadores sanitarios experimentaron trastornos del estado de ánimo y del sueño durante este brote, lo que enfatiza la necesidad de establecer formas de mitigar los riesgos para la salud mental y ajustar las intervenciones en condiciones de pandemia.

\section{Comentario}

Las infecciones de las vías respiratorias inferiores son las enfermedades transmisibles con mayor mortalidad en todo el mundo. En diciembre de 2019, surgió en Wuhan (China) un síndrome respiratorio agudo grave altamente infeccioso causado por un nuevo coronavirus (SARS-CoV-2), que recibió el nombre de COVID-19. La enfermedad fue declarada pandemia por la Organización Mundial de la Salud (OMS) en marzo de 2020. Ésta situación generó un estado excepcional en salud pública, con repercusiones en la salud mental de la población general y especialmente del personal sanitario ${ }^{(1,2)}$.

La aparición de enfermedades repentinas y potencialmente mortales, puede generar un gran estrés sobre los profesionales de la salud volviéndose estos especialmente vulnerables a los problemas de salud mental(3). En estas situaciones, los 
profesionales sanitarios pueden ver aumentada su carga de trabajo y su jornada laboral, sufrir un déficit de material de protección, tener miedo de contagiar a sus familiares y amigos, sentir aislamiento y discriminación social, así como atender a pacientes que se encuentran solos o mueren a consecuencia de la COVID-19(4).

Así, los profesionales sanitarios pueden presentar altos niveles de ansiedad, depresión, insomnio, burnout o incluso estrés postraumático ${ }^{(5-7)}$. Esta problemática puede afectar al ejercicio de sus funciones profesionales, disminuyendo sus capacidades de atención, comprensión y toma de decisiones, por lo que preservar la salud física, mental y social de los profesionales sanitarios resulta esencial en la lucha contra el virus ${ }^{(8)}$. Por todo ello es importante tener datos de alta calidad sobre los efectos sobre la salud mental de la pandemia de COVID-19 en toda la población y en grupos vulnerables como los profesionales de la salud(9).

El objetivo de esta revisión sistemática y metaanálisis analizados es examinar la evidencia emergente de los efectos del brote de COVID-19 en la salud mental de los trabajadores sanitarios y, en particular, en relación con la prevalencia de ansiedad, depresión e insomnio. El grupo de población del estudio son trabajadores de la salud (médicos y no médicos) en países afectados por la pandemia de COVID-19 (China y Singapur). Sólo los estudios que evaluaron las tasas de prevalencia de depresión, ansiedad o insomnio mediante métodos de evaluación validados fueron elegidos para su inclusión.

Esta revisión evidencia que una alta proporción de profesionales de la salud han experimentado niveles significativos de ansiedad, depresión e insomnio durante la pandemia de COVID-19. Las tasas de prevalencia de ansiedad y depresión (23.2\% y $22.8 \%$ respectivamente) de los trabajadores sanitarios durante el COVID-19 son comparables con las respectivas tasas reportadas para la población general en China durante el mismo período, lo que muestra el efecto considerable de la crisis en toda la población ${ }^{(10-13)}$.

El impacto en la salud mental del personal sanitario ya se había documentado durante los brotes de SARS-CoV-1 (2003), H1N1 (2009), MERS-CoV (2012) o ébola (2014), observándose niveles moderados y altos de ansiedad, depresión, estrés postraumático y absentismo. Los resultados en el caso de COVID-19 indican sintomatología más moderada que durante las crisis sanitarias de SARS y MERS, donde se observaron altas tasas de depresión y ansiedad. Las posibles diferencias entre estos brotes y la pandemia de COVID-19 podrían explicarse, por el potencial infeccioso extremadamente alto y la mayor tasa de mortalidad de estos y también por la experiencia ya adquirida. No obstante, la interpretación de estos datos iniciales debe realizarse con cautela, ya que en los brotes previos se ha demostrado la prolongación de los síntomas a medio y largo plazo, por lo que estos datos no son definitivos ${ }^{(14,15)}$.

Aunque las diferentes escalas y puntuaciones de corte adoptados por cada encuesta han podido introducir una gran heterogeneidad entre los estudios, parece que la mayoría de los trabajadores sanitarios experimentaron síntomas leves tanto de depresión como de ansiedad, mientras que los síntomas moderados y severos fueron menos comunes. Es por ello importante una detección temprana 
y el tratamiento de los síntomas clínicos más leves del estado de ánimo o los síndromes subumbrales antes de que evolucionen hacia respuestas psicológicas más complejas y duraderas ${ }^{(16)}$.

Por otro lado, el análisis de subgrupos reveló diferencias de grupo profesional, género y gravedad potencialmente importantes, sobre posibles vulnerabilidades particulares ${ }^{(17)}$. La tasa de prevalencia de ansiedad y depresión pareció ser más alta entre el personal de primera línea ${ }^{(18)}$. El personal de enfermería mostró estimaciones de prevalencia más altas tanto para la ansiedad como para la depresión en comparación con los médicos. No obstante, el impacto psicológico de la crisis no solo lo sufren los médicos y enfermeras de cuidados intensivos y respiratorios de primera línea, sino también el personal sanitario de otras especialidades ${ }^{(19)}$. También se observaron síntomas más frecuentes e intensos entre las mujeres, lo que probablemente refleja la brecha de género ya establecida para los síntomas de ansiedad y depresión ${ }^{(20)}$. Como se ha comentado, un hecho importante que merece consideración es la heterogeneidad inherente entre los estudios, de ahí el análisis de subgrupos por gravedad.

En el ámbito asiático, los estudios realizados, indicaron la necesidad de promover el bienestar psicológico de los profesionales. Se encontró que el nivel de apoyo social se correlacionaba positivamente con la autoeficacia y la calidad del sueño y negativamente con la ansiedad y el estrés. Una gran proporción de trabajadores sanitarios en Wuhan se vieron afectados y se observó que el apoyo en la salud mental era necesario incluso para reacciones psicológicas leves. Con este fin, se deben considerar intervenciones tempranas y específicas ${ }^{(21)}$.

Sin embargo, se desconoce si estos planes y medidas de apoyo son aplicables en otros ámbitos geográficos y culturales, por este motivo es importante explorar los efectos psicológicos de la COVID-19 en el ámbito occidental, a fin de conocer los riesgos asociados y posibles factores de protección, para poder diseñar estrategias eficientes de apoyo y ayuda para la salud mental(22).

Por otro lado, ante el mayor riesgo y exposición de las mujeres a los problemas de salud mental, tanto en general como en situaciones de pandemia, y teniendo en cuenta la alta feminización del sector sanitario, las intervenciones y medidas de apoyo para disminuir el impacto psicológico, tendrían que incluir la perspectiva de género(23).

Puesto que la mayoría de los estudios se realizaron en China, la generalización de los hallazgos puede ser limitada, ya que los sistemas de salud varían mucho entre países. No obstante, teniendo en cuenta que China se vio gravemente afectada, los resultados proporcionan una indicación fiable del potencial de la pandemia de afectar la salud mental de los trabajadores sanitarios.

En conclusión, esta revisión sistemática y metaanálisis proporcionan una síntesis de la evidencia existente que destaca las altas tasas de prevalencia de depresión, ansiedad e insomnio de los profesionales de la salud. Los resultados pueden ayudar a cuantificar las necesidades de apoyo del personal y crear intervenciones escalonadas y personalizadas en condiciones de pandemia que mejoren la resiliencia y mitiguen la vulnerabilidad del personal sanitario(24,25). 


\section{Referencias}

1. Dong L, Bouey J, Bouey J. Public Mental Health Crisis during COVID-19 Pandemic, China. Emerg Infect Dis. 2020;26:1616-1618.

2. Xiong J, Lipsitz O, Nasri F, Lui LMW, Gill H, Phan L et al. Impact of COVID-19 pandemic on mental health in the general population: A systematic review. J Affect Disord. 2020;277:55-64.

3. Serrano-Ripoll MJ, Meneses-Echavez JF, Ricci-Cabello I, Fraile-Navarro D, Fiol-deRoque MA, Pastor-Moreno G et al. Impact of viral epidemic outbreaks on mental health of healthcare workers: a rapid systematic review and meta-analysis. J Affect Disord. 2020;277:347-357.

4. Kunzler AM, Röthke N, Günthner L, Stoffers-Winterling J, Tüscher O, Coenen M et al. Mental burden and its risk and protective factors during the early phase of the SARS-CoV-2 pandemic: systematic review and meta-analyses. Global Health. $2021 ; 17$.

5. Shaukat N, Ali DM, Razzak J. Physical and mental health impacts of COVID-19 on healthcare workers: A scoping review. Int J Emerg Med. 2020;13:1-8.

6. Li Y, Scherer N, Felix L, Kuper H. Prevalence of depression, anxiety and posttraumatic stress disorder in health care workers during the COVID-19 pandemic: A systematic review and meta-Analysis. PLoS One. 2021;16:e0246454.

7. da Silva FCT, Neto MLR. Psychiatric symptomatology associated with depression, anxiety, distress, and insomnia in health professionals working in patients affected by COVID-19: A systematic review with meta-analysis. Prog Neuropsychopharmacology Biol Psychiatry. 2021;104:110057.

8. Guo J, Liao L, Wang B, Li X, Guo L, Tong Z et al. Psychological Effects of COVID-19 on Hospital Staff: A National Cross-Sectional Survey of China Mainland. SSRN Electron J. 2020. Disponible en: https://papers.ssrn.com/sol3/papers. cfm?abstract_id $=3550050$

9. Huang $Y$, Zhao N. Generalized anxiety disorder, depressive symptoms and sleep quality during COVID-19 outbreak in China: a web-based cross-sectional survey. Psychiatry Res. 2020;288:112954.

10. Wang C, Pan R, Wan X, Tan Y, Xu L, Ho CS et al. Immediate psychological responses and associated factors during the initial stage of the 2019 coronavirus disease (COVID-19) epidemic among the general population in China. Int J Environ Res Public Health. 2020;17:1729.

11. Wang $C$, Pan $R$, Wan $X$, Tan $Y, X u L$, Mclntyre RS et al. A longitudinal study on the mental health of general population during the COVID-19 epidemic in China. Brain Behav Immun. 2020;87:40-48.

12. Arora T, Grey I, Östlundh L, Lam KBH, Omar OM, Arnone D. The prevalence of psychological consequences of COVID-19: A systematic review and meta-analysis of observational studies. J Health Psychol. 2020;29:135910532096663. 
13. Luo M, Guo L, Yu M, Wang H. The psychological and mental impact of coronavirus disease 2019 (COVID-19) on medical staff and general public - A systematic review and meta-analysis. Psychiatry Res. 2020;291:113190.

14. De Brier N, Stroobants $S$, Vandekerckhove P, De Buck E. Factors affecting mental health of health care workers during coronavirus disease outbreaks (SARS, MERS \& COVID-19): A rapid systematic review. PLoS One. 2020;15(12):e0244052.

15. Cabarkapa S, Nadjidai SE, Murgier J, Ng CH. The psychological impact of COVID-19 and other viral epidemics on frontline healthcare workers and ways to address it: A rapid systematic review. Brain Behav Immun Heal. 2020;8:100144.

16. Vizheh M, Qorbani M, Arzaghi SM, Muhidin S, Javanmard Z, Esmaeili M. The mental health of healthcare workers in the COVID-19 pandemic: A systematic review. J Diabetes Metab Disord. 2020;19:1967-1978.

17. Lai J, Ma S, Wang Y, Cai Z, Hu J, Wei $N$ et al. Factors associated with mental health outcomes among health care workers exposed to coronavirus disease 2019. JAMA Netw Open. 2020;3:203976.

18. Salari N, Khazaie H, Hosseinian-Far A, Khaledi-Paveh B, Kazeminia M, Mohammadi $\mathrm{M}$ et al. The prevalence of stress, anxiety and depression within front-line healthcare workers caring for COVID-19 patients: a systematic review and meta-regression. Hum Resour Health. 2020;18:100.

19. Al Maqbali M, Al Sinani M, Al-Lenjawi B. Prevalence of stress, depression, anxiety and sleep disturbance among nurses during the COVID-19 pandemic: A systematic review and meta-analysis. J Psychosom Res. 2021;141:110343.

20. Albert PR. Why is depression more prevalent in women? J Psychiatry Neurosci. $2015 ; 40: 219-221$.

21. Soklaridis $S$, Lin E, Lalani Y, Rodak T, Sockalingam S. Mental health interventions and supports during COVID- 19 and other medical pandemics: A rapid systematic review of the evidence. Gen Hosp Psychiatry. 2020;66:133-146.

22. Holmes EA, O'Connor RC, Perry VH, Tracey I, Wessely S, Arseneault L et al. Multidisciplinary research priorities for the COVID-19 pandemic: a call for action for mental health science. Lancet Psychiatry. 2020;7:547-560.

23. López-Atanes $M$, Recio-Barbero $M$, Sáenz-Herrero $M$. Are women still "the other"? Gendered mental health interventions for health care workers in Spain during COVID-19. Psychol Trauma. 2020;12:S243-S244.

24. Pollock A, Campbell P, Cheyne J, Cowie J, Davis B, McCallum J et al. Interventions to support the resilience and mental health of frontline health and social care professionals during and after a disease outbreak, epidemic or pandemic: a mixed methods systematic review. Cochrane Database Syst Rev. 2020;11(11):CD013779.

25. Kisely S, Warren N, McMahon L, Dalais C, Henry I, Siskind D. Occurrence, prevention, and management of the psychological effects of emerging virus outbreaks on healthcare workers: rapid review and meta-analysis. BMJ. 2020;369:m1642. 